Modern Physics Letters A

(C) World Scientific Publishing Company

\title{
SEARCHES FOR LONG-LIVED PARTICLES AT THE TEVATRON COLLIDER
}

\author{
TODD ADAMS \\ Department of Physics, Florida State University, 515 Keen Building \\ Tallahassee, Florida 32306, U.S.A. \\ tadams@hep.fsu.edu
}

Received (Day Month Year)

Revised (Day Month Year)

\begin{abstract}
Several searches for long-lived particles have been performed using data from $p \bar{p}$ collisions from Run II at the Tevatron. In most cases, new analysis techniques have been developed to carry out each search and/or estimate the backgrounds. These searches expand the discovery potential of the CDF and D0 experiments to new physics that may have been missed by traditional search techniques. This review discusses searches for (1) neutral, long-lived particles decaying to muons, (2) massive, neutral, long-lived particles decaying to a photon and missing energy, (3) stopped gluinos, and (4) charged massive stable particles. It summarizes some of the theoretical and experimental motivations for such searches.

Keywords: searches; long-lived; supersymmetry.

PACS Nos.: 13.85.Rm, 14.80.Ly, 14.80.Cp.
\end{abstract}

\section{Introduction}

The discovery of new particle phenomena has led to some of the greatest advances in our understanding of particle physics, particularly when the discovery is unexpected. Among the most famous are the muon (1937), strange mesons ("V" particles, 1947), the $J / \psi$ meson (1974), and the tau lepton (1974). There is broad agreement among particle physicists that progress in understanding some fundamental questions, such as the origin of mass, requires new physics. While there are many interesting theories of possible new physics, a few of which we shall touch upon in this review, no one actually knows what Nature has in store for us. Therefore, it is imperative that modern particle physics experiments search for signs of new physics in as many different ways as possible, including those that are motivated by current theory and those that are not.

Numerous searches for new particle phenomena have been done using data from the Tevatron, the Fermi National Accelerator Laboratory (Fermilab) hadron collider. Most of these searches focus on reconstructing particles that decay within millimeters of the beamline or on observing missing transverse energy signatures 
from stable particles that escape without interacting. However, as is shown in this review, the multipurpose design of the Tevatron detectors, D0 and CDF, allows a broader range of searches to be conducted, namely for long-lived, but unstable particles. Many possibilities exist that give rise to unique signatures.

\subsection{Theoretical Motivation}

A wide variety of theoretical models, including large classes based on supersymmetry (SUSY), allow for long-lived particles. The simplest SUSY variation is $R$-parity violation which allows the lightest supersymmetric particle (LSP) to decay purely to non-SUSY particles. ${ }^{1}$ The rate of such decays is generally determined by one or more couplings. Numerous searches at both the LEP and Tevatron colliders have looked for (nearly) prompt $R$-parity violating processes, however they are insensitive to longer lifetimes where the coupling is small.2|3|4|5|6|7

Gauge mediated supersymmetry breaking (GMSB) models, in which the supersymmetry breaking is mediated by gauge fields other than gravity, generally have a light $(\mathrm{MeV})$ gravitino LSP and all other supersymmetric particles decay via cascades to the next-to-lightest supersymmetric particle (NLSP). The coupling to gravity can determine how quickly the NLSP decays to its non-SUSY partner and the gravitino. If the symmetry breaking scale is small (larger coupling), the lifetime is short. Conversely, if the scale is large, this results in a long-lived NLSP that can decay either within the detector or beyond it 6 Favored NLSP candidates include the neutralino $\left(\chi_{1}^{0}\right)$ or the stau $(\tilde{\tau})$.

Long lifetimes can also arise from small mass splittings between the NLSP and the LSP 9 If the mass difference $\left(M_{N L S P}-M_{L S P}\right)$ is small enough, phase space suppression will impede the decay of the NLSP. One particular model of interest here is inspired by anomaly mediated supersymmetry breaking (AMSB) with a chargino NLSP and a mass splitting $<150 \mathrm{MeV}$.

Split supersymmetry, where SUSY scalars are much heavier than SUSY fermions, can suppress the decay rate of gluinos giving them long lifetimes $10 \mid 11$ They may live long enough to hadronize into "R-hadrons," colorless, bound states of the gluino, quarks, and gluons 12 Nuclear interactions can cause some neutral R-hadrons to become charged, leading to a number of interesting signatures within the detector. 13

There has been recent discussions of a class of models referred to as "hidden valley" models which can yield new phenomena resulting in long-lived particles through reduced coupling to standard model (SM) particles. 14|15|16 The hidden valley structures are very general and can be added to many beyond the standard model (BSM) theories. Most interestingly, they can give rise to significantly different phenomenology from that commonly associated with the original BSM model ${ }^{14} \mathrm{An}$ example is a light Higgs boson that decays to two neutral, long-lived particles that further decay to $b$-jets. 15 These "hidden valley" models provide a timely cautionary tale: if one only looks where the light shines brightest, one risks missing a possible spectacular prize in the shadows. 


\subsection{Experimental Motivation}

The $\mathrm{NuTeV}$ experiment at Fermilab observed an unexpected excess of events in one of three searches for long-lived neutral particles. 17 The NuTeV neutrino detector was augmented with a $30 \mathrm{~m}$ long, low-mass decay region in front. The decay region was instrumented with several drift chambers along its length. A weakly-interacting, long-lived neutral particle produced by proton-nucleon interactions from the 800 $\mathrm{GeV}$ Tevatron beam could travel $1.4 \mathrm{~km}$ and decay in the low mass region.

$\mathrm{NuTeV}$ performed three separate searches (in mass bins) by reconstructing a vertex from a pair of tracks from hits in the decay region and using particle identification from the neutrino detector. In the high mass $(2.2-15 \mathrm{GeV})$ search, three events were reconstructed with a vertex from two muons and an unobserved neutrino. The largest backgrounds originate from neutrino scattering within the decay region and in the upstream shielding. The total estimated background was $0.07 \pm$ 0.01 events, which is to be compared with three observed events.

No widely accepted explanation of this excess has been found. No backgrounds are large enough to explain it. Cross-checks performed with events of similar topology within the neutrino detector support the hypothesis that the events are unlikely to be due to neutrino scattering. If a new particle is responsible, the three events are consistent with a particle of $\approx 5 \mathrm{GeV}$ mass decaying to $\mu^{+} \mu^{-} \nu$. The best theoretical hypothesis for a new particle is a long-lived neutralino from $B$ mesons produced at the primary proton target $\frac{18}{18}$ Unfortunately, no current experiment matches NuTeV's running conditions so it has been impossible to repeat the same search. However, as detailed below, collider experiments could, in principle, observe such a hypothetical particle, depending on the production mechanism.

\subsection{The CDF and DO Detectors}

While the specific detectors differ in technology and implementation, the CDF 19 and $\mathrm{D} 0^{20}$ detectors share similar general characteristics. The overall structure (from inside outward) consists of a central tracking region, a calorimeter, and a muon system (see Fig. 1 for a generic schematic). The central tracking system consists of a silicon tracking detector surrounded by another large volume tracker within a solenoid magnet. The silicon tracker provides precision vertex measurement as well as identification of heavy quarks using secondary vertices. The outer tracker, with its large lever arm, measures the transverse momenta of charged tracks. Table 1 lists some of the specific characteristics of the individual detectors.

Collider detectors are optimized to reconstruct particles that decay within millimeters of the beamline (including bottom or charm quarks) or to detect stable particles that escape without interacting (e.g. neutrinos) through the measurement of missing transverse energy. However, they can also be used to search for particles that decay with long lifetimes. Below we review several analyses that use techniques including detached vertex reconstruction, timing, and unique signatures to search for such particles. 


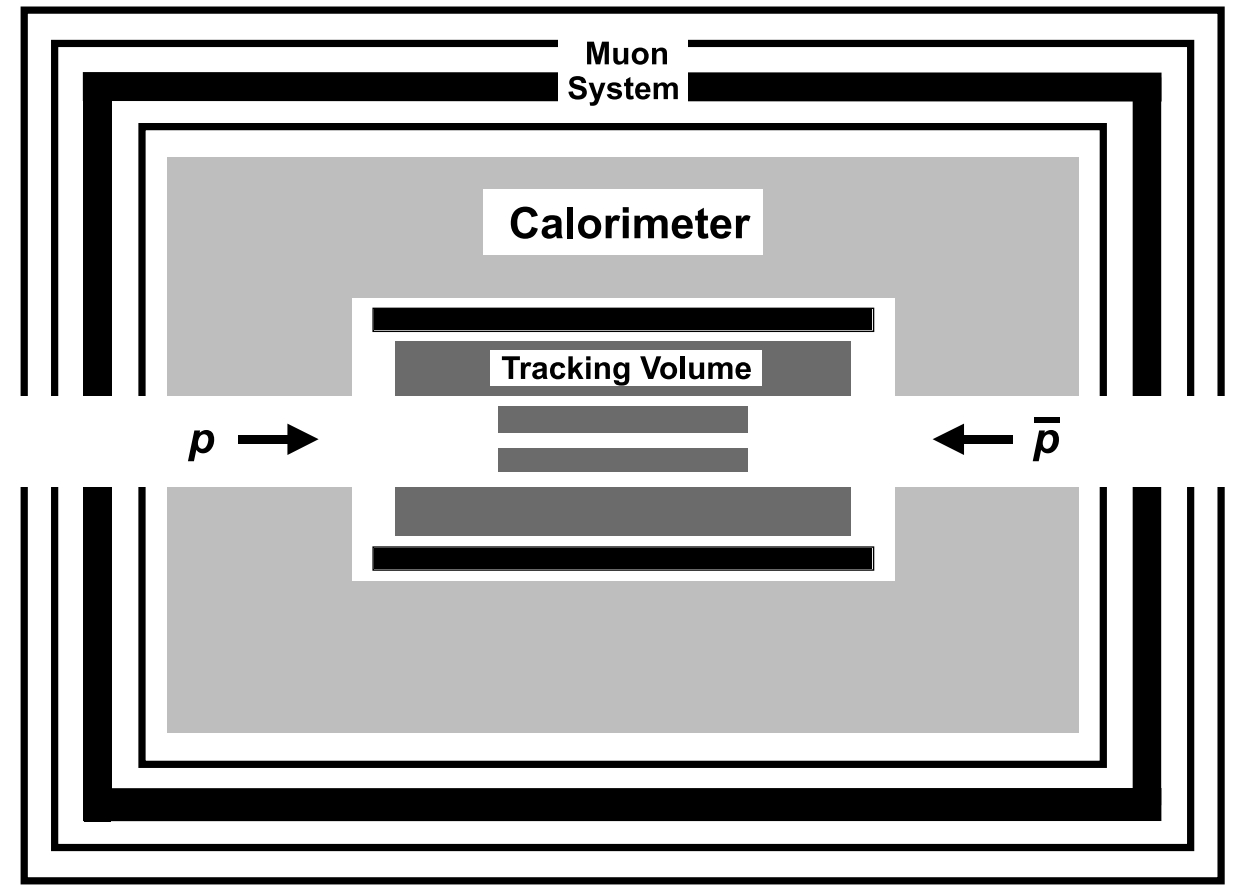

Fig. 1. A general systematic (side view) of the Tevatron collider detectors. The interaction region is at the center. The gray indicates tracking volumes, the black boxes show solenoid (inner) and toroid (outer) magnets, the light gray region represents the calorimeter, and the black lines show the muon system.

Table 1. Summary of CDF and D0 detector technologies and characteristics.

\begin{tabular}{lcc} 
& CDF & D0 \\
\hline Inner Tracker & silicon & silicon \\
Outer Tracker & drift chamber & fiber tracker \\
Tracking Volume Radius & $1.3<r<132 \mathrm{~cm}$ & $1.5<r<50 \mathrm{~cm}$ \\
Solenoid Magnet & $1.4 \mathrm{~T}$ & $2 \mathrm{~T}$ \\
Calorimeter & iron/scintillator & liquid-Ar/Uranium \\
Muon System & drift tubes and scintillator & drift tubes and scintillator \\
Muon Toroid Magnet & $1.4 \mathrm{~T}$ & $1.8 \mathrm{~T}$ \\
\hline
\end{tabular}

\section{Neutral Long-lived Particles Decaying to Two Muons}

D0 has carried out a search for neutral particles that have a (relatively) long lifetime and decay to two muons plus missing transverse energy 21 One of the motivations for this search was to explore what the Tevatron collider can say about the $\mathrm{NuTeV}$ result described in Sec. 1.2. The signature was a highly displaced vertex reconstructed using tracks in the central fiber tracker (CFT), which are matched to signals in the muon system. The neutral particle must travel at least 5-20 $\mathrm{cm}$ in the transverse 
plane before decaying. These decay lengths are significantly longer than those of normal $b$-hadrons. Studies of $K_{S} \rightarrow \pi^{+} \pi^{-}$decays demonstrated the ability to find these highly displaced vertices.

Events with two isolated muons with $p_{T}>10 \mathrm{GeV}$ were selected. The muons were required to have a distance of closest approach (DCA) to the primary interaction vertex (PV) of greater than $0.01(0.1) \mathrm{cm}$ in the $\mathrm{x}-\mathrm{y}$ plane (along the $z$ axis). This reduces events where the muons originate from the PV. The muon tracks were fit to a common vertex and its distance in the transverse dimension (i.e. the radius) was calculated from $r=\sqrt{\left(X-X_{P V}\right)^{2}+\left(Y-Y_{P V}\right)^{2}}$ where $X_{P V}, Y_{P V}$ are the $x$ and $y$ coordinates of the primary vertex. The final event selection requires $5<r<20 \mathrm{~cm}$.

There are no SM sources of highly displaced vertices involving muon pairs, so the largest backgrounds originate from mis-reconstruction. Such instrumental backgrounds are typically difficult to model accurately, therefore, D0 estimated the background from data that closely (but not exactly) resemble those in the final selection. Events with $0.3<r<5 \mathrm{~cm}$ and/or only one muon passing the DCA requirement were used. The total background was estimated to be $0.75 \pm 1.5$ events.

No events were found to pass all of the selection criteria in data, therefore, limits on the production cross section times branching ratios were set (Fig. 2). The limit depends on the lifetime and the mass (through the acceptance). A comparison was made with limits set by $\mathrm{NuTeV}$ as well as with what one would expect if the $\mathrm{NuTeV}$ excess is interpreted as a signal. To do so, the $\mathrm{NuTeV}$ limit was converted from that of $p p$ interactions at $\sqrt{s}=38 \mathrm{GeV}$ to one for $p \bar{p}$ interactions at $\sqrt{s}=1960 \mathrm{GeV}$ using a model of neutralino pair production in an unconstrained minimal supersymmetric model (uMSSM) $\frac{22}{2}$ As a result, D0 can exclude some interpretations of the $\mathrm{NuTeV}$ result at all lifetimes.

\section{Neutral Long-lived Particles Decaying to Photons}

CDF performed a search for a heavy, long-lived particle decaying to a photon and an unobserved particle .23 A heavy particle produced at the Tevatron would move slowly away from the interaction point. If the particle is unstable, yet long-lived, its decay products would be delayed in reaching the various detectors relative to those from prompt decays ${ }^{24} \mathrm{CDF}$ implemented measurement of the timing of particles arriving at its electromagnetic calorimeter with a timing resolution of $0.6 \mathrm{~ns} . \frac{25 \mid 26}{25}$

The timing measurement formed the basis of a search for delayed photons from decays of massive particles. While the search is model independent, the prototypical GMSB signal $\chi_{1}^{0} \rightarrow \gamma G$, with a $\chi_{1}^{0}$ lifetime of 5 ns was used to estimate a typical acceptance for such events. Events were selected with at least one high $p_{T}(>30 \mathrm{GeV})$ photon, one jet (which could also be from a photon), and large missing transverse energy $(>30 \mathrm{GeV})$.

The corrected arrival time of the photon $\left(t_{c}^{\gamma}\right)$ is defined as $t_{c}^{\gamma}=t_{f}-t_{i}-\frac{\left|\vec{x}_{f}-\vec{x}_{i}\right|}{c}$ where $t_{i, f}$ and $\vec{x}_{i, f}$ are the initial and final photon time and position. The initial 

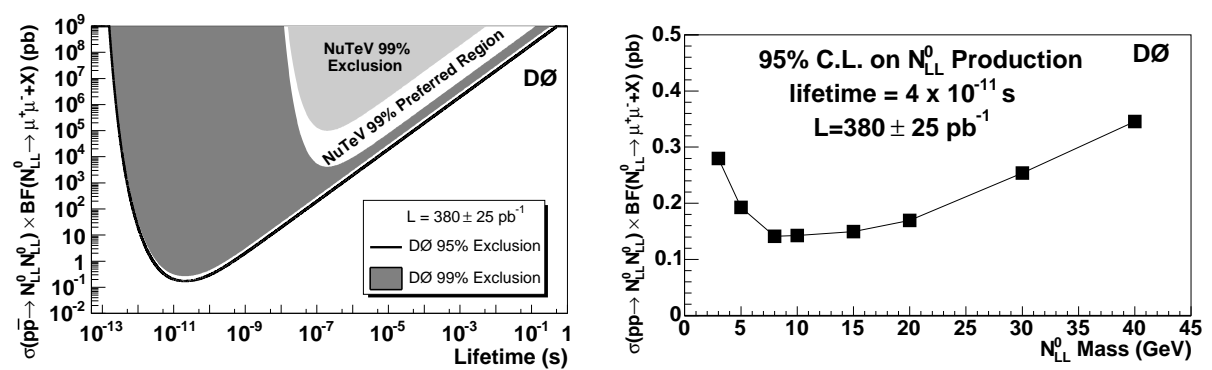

Fig. 2. (left) The limit on the pair production cross section times branching ratio for a $5 \mathrm{GeV}$ neutral, weakly-interacting particle that decays to two muons and an unobserved particle as a function of the particle lifetime. The published $\mathrm{NuTeV}$ limit is converted to the D0 running conditions using an uMSSM production model. The light band indicates the region favored by an interpretation of the three observed events as signal. (right) The limit as a function of particle mass for a lifetime of $4 \times 10^{-11}$ seconds. Reprinted figures with permission from V. M. Abazov et al. [D0 Collaboration], Phys. Rev. Lett. 97, 161802 (2006). Copyright 2006 by the American Physical Society.

position is given by the primary vertex while the final position is the arrival at the calorimeter. Photons produced directly or via prompt decays have a Gaussian time distribution with a mean of zero. The analysis searches for an excess of events with $2<t_{c}^{\gamma}<10 \mathrm{~ns}$.

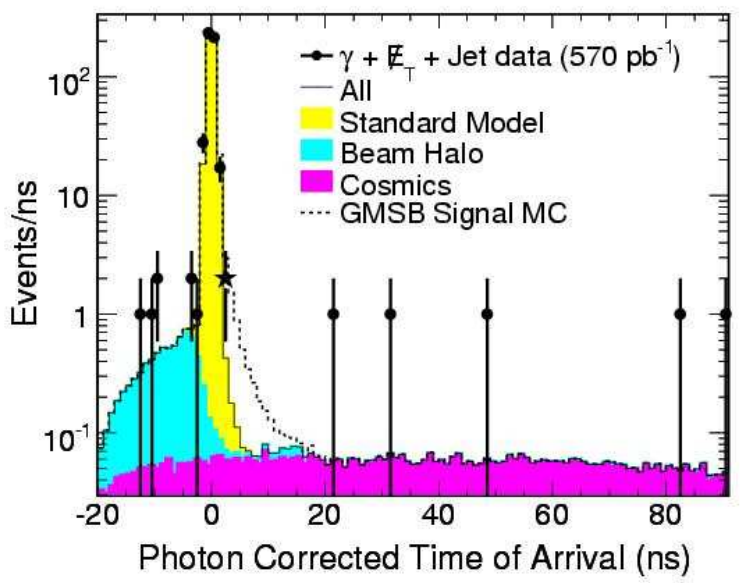

Fig. 3. The corrected arrival time of the photon $\left(t_{c}^{\gamma}\right)$ for data (black points), SM backgrounds (yellow), beam halo (blue), and cosmics (magenta). Sample GMSB signal is shown as the dashed line. The signal region corresponds to $t_{c}^{\gamma}=2-10 \mathrm{~ns}$. Reprinted figure with permission from A. Abulencia et al. [CDF Collaboration], Phys. Rev. Lett. 99, 121801 (2007). Copyright 2007 by the American Physical Society. 

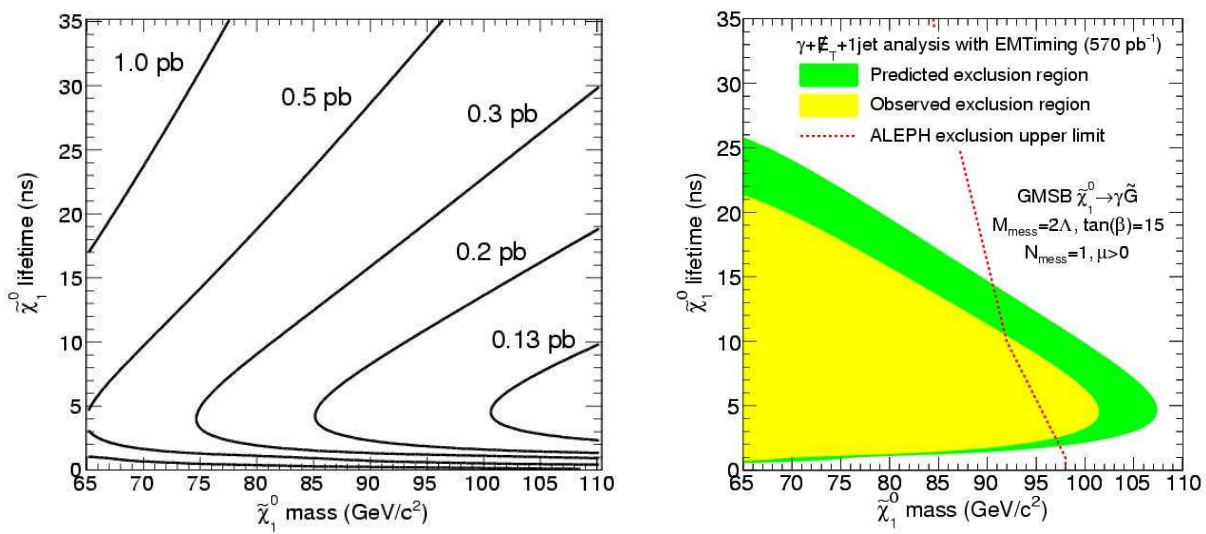

Fig. 4. (left) $95 \%$ CL cross section limit contours as a function of the $\chi_{1}^{0}$ lifetime vs. mass. (right) Expected and excluded regions of GMSB parameter space as a function of $\chi_{1}^{0}$ lifetime vs. mass. Reprinted figures with permission from A. Abulencia et al. [CDF Collaboration], Phys. Rev. Lett. 99, 121801 (2007). Copyright 2007 by the American Physical Society.

Standard model backgrounds from collisions include $\gamma+$ jet, dijet, and $W \rightarrow e \nu$ events. In the first two cases, the missing transverse energy must arise from energy mis-measurement. In dijet $(W)$ events, a jet (electron) is mis-identified as a photon. Non-SM backgrounds include cosmic ray muons and beam halo muons with a photon from bremsstrahlung. Figure 3 shows distributions of the corrected arrival time for each background, the data, and for a sample signal. All background normalizations were determined by fitting data in regions of $t_{c}^{\gamma}$ outside of the signal region. Signal acceptance was estimated using Monte Carlo simulations.

After optimization of several selection criteria, background was estimated to be $1.25 \pm 0.66$ events $(0.71 \pm 0.60$ from SM, $0.46 \pm 0.26$ from cosmics, $0.07 \pm 0.05$ from beam halo). Two events were observed in data. The signal acceptance depends on the mass of the long-lived particle as well as its lifetime. Therefore, limits on the production cross section are set in the lifetime versus mass plane (Fig. (4). For the specific GMSB model used to model the signal, the limits set by CDF in the high mass region are greater than those from a previous analysis by ALEPH (see Ref. 27).

\section{Stopped Particles}

D0 has carried out a search for very long-lived, heavy particles which stop within the calorimeter and decay microseconds to days later ${ }^{28}$ As mentioned in Sec. 1.1, split supersymmetry provides a plausible candidate. In one scenario, charged R-hadrons lose energy via ionization and some fraction can stop within the calorimeter (called stopped gluinos). At some later time the gluino can decay to a gluon and a neutralino 

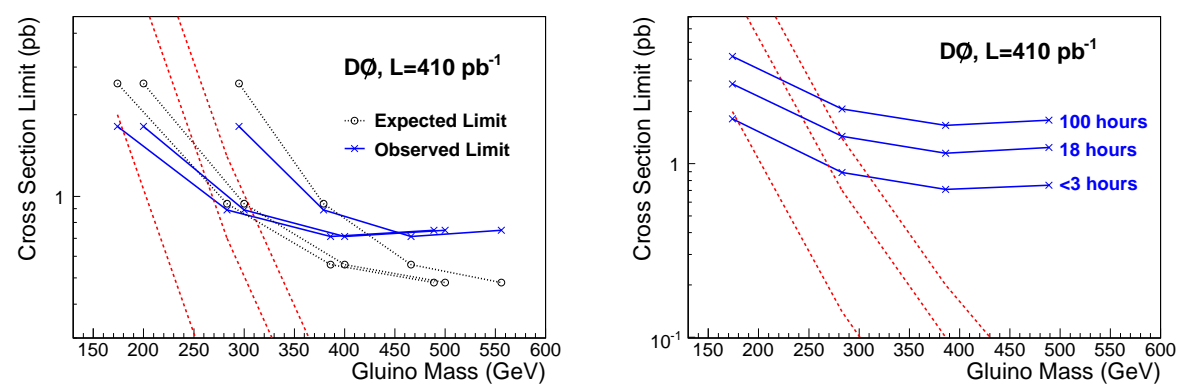

Fig. 5. Cross section limits on stopped gluinos. (left) Limits assuming a lifetime $<3$ hours, $100 \%$ BR to $g \chi_{1}^{0}$, and three different $\chi_{1}^{0}$ masses $(50,90$, and $200 \mathrm{GeV}$, left to right). The expected limit is shown in black and the observed limit in blue. The red lines indicate theoretical cross sections for a range of assumed conversion cross sections. (right) Limits for a $50 \mathrm{GeV} \chi_{1}^{0}$ mass for three different gluino lifetimes ( $<3$ hours, 18 hours, and 100 hours). Reprinted figures with permission from V. M. Abazov et al. [D0 Collaboration], Phys. Rev. Lett. 99, 131801 (2007). Copyright 2007 by the American Physical Society.

$\left(G \rightarrow g \chi_{1}^{0}\right)$ or a quark-antiquark and a neutralino $\left(G \rightarrow q \bar{q} \chi_{1}^{0}\right)$ giving a large energy deposition in the calorimeter that is not associated (in time) with the interaction that produced it.

For such events, one expects a large energy deposit in the calorimeter associated with large missing transverse energy and no other significant event activity. However, because of trigger limitations, the analysis is only sensitive to gluino decays that occur during (or just before) a bunch crossing. Events were therefore selected that have no activity in luminosity counters (located near the beamline) indicative of no $p \bar{p}$ interaction.

Backgrounds are dominated by fake signals from cosmic muons and beam halo muons. The selection criteria have reduced the backgrounds from $p \bar{p}$ interactions to a negligible level. Fakes from cosmic and halo muons were estimated from complimentary data samples. Multiple searches were performed in bins of energy deposition in the calorimeter. It was found that in all cases the data were consistent with estimated backgrounds. The signal sensitivity depends on the gluino lifetime and on the mass of the LSP $\left(\chi_{1}^{0}\right)$. Figure 5 shows the cross section limit for gluino production as a function of gluino mass for different $\chi_{1}^{0}$ masses and gluino lifetimes. These limits are generally model-independent and apply to any long-lived monojet signal.

\section{Charged Massive Stable Particles}

The searches discussed above focused on neutral particles that decay to stable particles within the detector. However, new long-lived, charged particles are also possible. If the lifetime is short enough to decay within the central tracking system, the most likely signature is a charged track which has a kink (or additional tracks) 
in the middle of it. These topologies have been searched for at LEP 29 New stable, charged particles are limited by cosmological constraints to masses below 230 $\mathrm{GeV} \stackrel{30}{ }$ In addition, the observation and measurement of the mass/lifetime of a long-lived, charged particle could have implications on understanding of Big Bang nucleosynthesis. .31

However, if the particle lives long enough to pass through the detector, it can leave other striking signatures. A massive particle will be slower moving than the typical stable SM particle and will leave behind a greater ionization trail (due to the effect of the particle mass in the Bethe-Bloch formula). If it does not interact strongly, the particle will look like a slow moving, heavily ionizing muon $\stackrel{32}{3}$ Both CDF and D0 have analyses underway (with preliminary results available and discussed below) that use timing techniques to look for such particles. A previous Run I analysis by CDF used a $d E / d x$ technique ${ }^{33}$ A review of the theoretical issues and experiment status of searches for massive, stable particles is available in Ref. 34 .

\subsection{CDF CHAMPs Search}

CDF has performed a search for CHArged Massive Particles (CHAMPs) using the timing capabilities of its outer tracker. ${ }^{35}$ Events are selected with at least one muon (with $p_{T}>20 \mathrm{GeV}$ ) that fired a single muon trigger and is from the primary vertex. Cosmic ray muons are rejected by looking for a second track where the pair is consistent with one track moving inward and the other moving outward. CHAMP candidate tracks include the trigger muon and either a second muon or the leading non-muon. Two samples are used: the signal sample where both candidates have $p_{T}>40 \mathrm{GeV}$ and a control sample where both have $20<p_{T}<40$.

For each CHAMP candidate, its velocity was measured from its path length and the time it arrives at the time-of-flight (TOF) detector (part of the tracking system). Muons have a velocity $\beta=v / c=1$ while massive particles have $\beta<1$. The momentum and velocity were used to calculate the candidate mass. $W \rightarrow e \nu$ events were used to study the efficiencies and the ability to model backgrounds in the signal sample using the control sample. Figure 6 shows the observed and predicted mass distribution for the signal sample. The region $M>100 \mathrm{GeV}$ is the final CHAMP sample.

The sample model used to estimate acceptance involved pair production of longlived stop squarks. $\frac{36}{36}$ The stop hadronizes (similar to the gluinos described in Sec. 4) and hadronic effects such as charge exchange were taken into account. Only one candidate CHAMP was found with $M>100 \mathrm{GeV}$, consistent with the predicted background. Figure 7 shows the CDF preliminary limit on the stable, massive, stop production cross section. From the calculated next-to-leading order (NLO) cross section, a limit on the stop mass $>240 \mathrm{GeV}$ was set. 


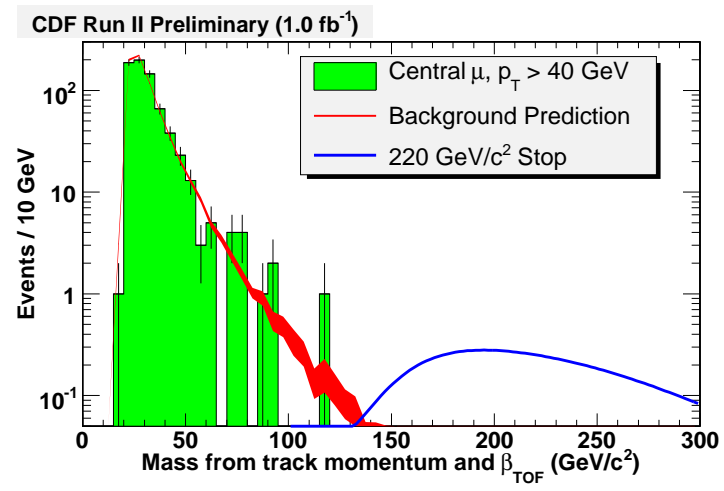

Fig. 6. Mass distribution for signal events calculated using the TOF detector. The filled histogram shows data while the red line indicates the expectation from the control region. The blue line indicates the expectation for a $220 \mathrm{GeV}$ stop mass.

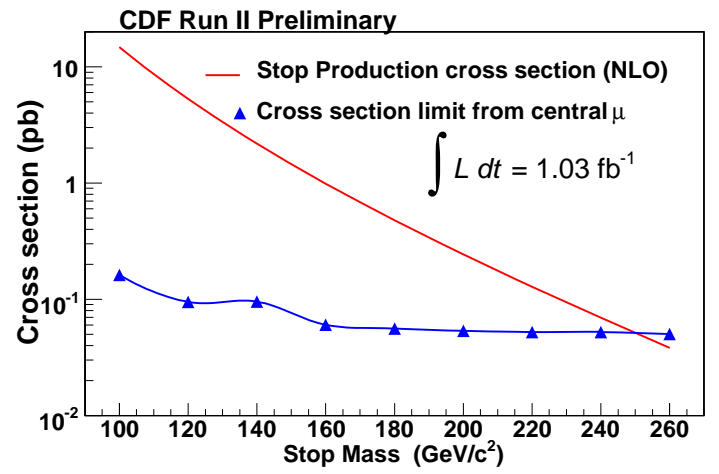

Fig. 7. Observed limit on stop production cross section (blue line with points) as a function of mass. The red line shows the NLO production cross section.

\subsection{DO CMSP Search}

D0 labels its search as CMSP - charged, massive, stable particles $\frac{37}{3 t}$ uses two different signal models as possible sources of CMSPs. The first is a GMSB model with the $\tilde{\tau}$ as the NLSP. Stau pair production is modeled with a lifetime sufficiently large that both $\tilde{\tau}$ 's decay outside the detector $[8$ The second model is an anomalymediated supersymmetry breaking (AMSB) inspired model where the LSP $\left(\chi_{1}^{0}\right)$ and NLSP $\left(\chi_{1}^{ \pm}\right)$mass difference is sufficiently small $(<150 \mathrm{MeV})$ to provide a long lifetime to the $\chi_{1}^{ \pm} ! 9$ Two chargino cases are considered: (1) where the chargino is mostly higgsino and (2) where it is mostly wino.

The analysis searches for pair production of long-lived staus or charginos having 

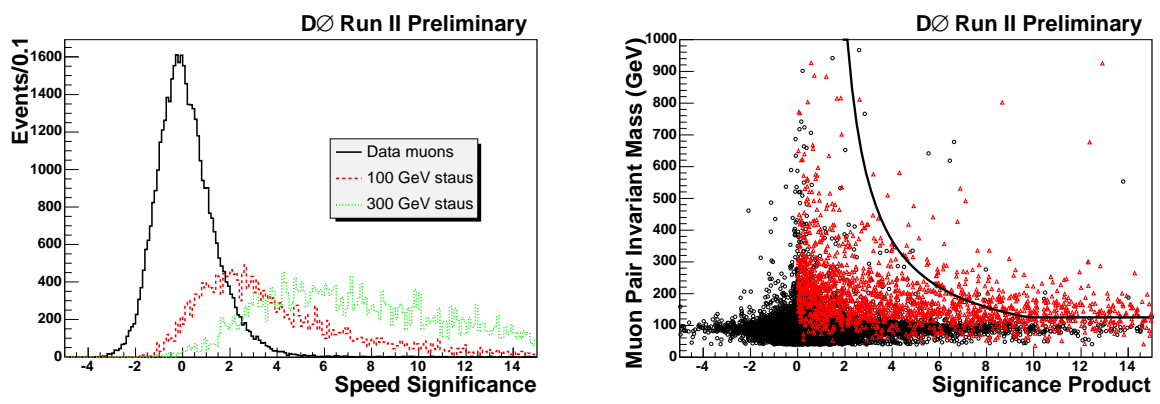

Fig. 8. (left) The speed significance for data (black) and two different stau masses (red and green). (right) Distribution of the invariant mass of the muon pair versus the speed significance product. The black dots indicate data, the red triangles show a $60 \mathrm{GeV}$ stau, while the black line shows a sample final analysis cut in this parameter space.

two CMSPs per event. Therefore, events are selected that contain two muons, which are generally back-to-back in $\phi$. For each muon, the average speed was calculated using the timing information from each of the three layers of the muon scintillator system. A muon travels at the speed of light while a massive particle will move slower. The sensitivity was therefore limited at lower masses by the timing resolution and at higher masses by the trigger that only accepted muons within its timing window.

The average speed of the particle is used to calculate the speed significance:

$$
\text { Significance }=\frac{1-\text { speed }}{\sigma_{\text {speed }}},
$$

where speed is measured with respect to the speed of light and $\sigma_{\text {speed }}$ is the uncertainty on the speed measurement due to timing resolution. Muons will have a significance centered at zero while massive particles are expected to have a large positive value (Fig. 8 (left)). Events are required to have both muons with positive speed significance. The final analysis cut used the speed significance product (the product of the two individual significances) and the invariant mass of the two muons. By cutting in this two dimensional space, background was greatly reduced while retaining signal events (Fig. 8(right)). This final criterion was optimized for six $\tilde{\tau}$ signal masses: $60,100,150,200,250$, and $300 \mathrm{GeV}$.

For all six masses, the observed data were consistent with background, therefore preliminary limits were set on the $\tilde{\tau}$ pair production cross section (Fig. 9(left)). However, the analysis is not yet sensitive to exclude masses beyond the LEP limit. Because the kinematics for chargino production are similar, the same cuts were applied and preliminary limits set on the chargino pair production cross section (Fig. 9(right)). Here, D0 excluded long-lived charginos with a mass below $174 \mathrm{GeV}$. However, a shorter lifetime (such that some fraction of the charginos decay before reaching the muon system) will lower this limit. 

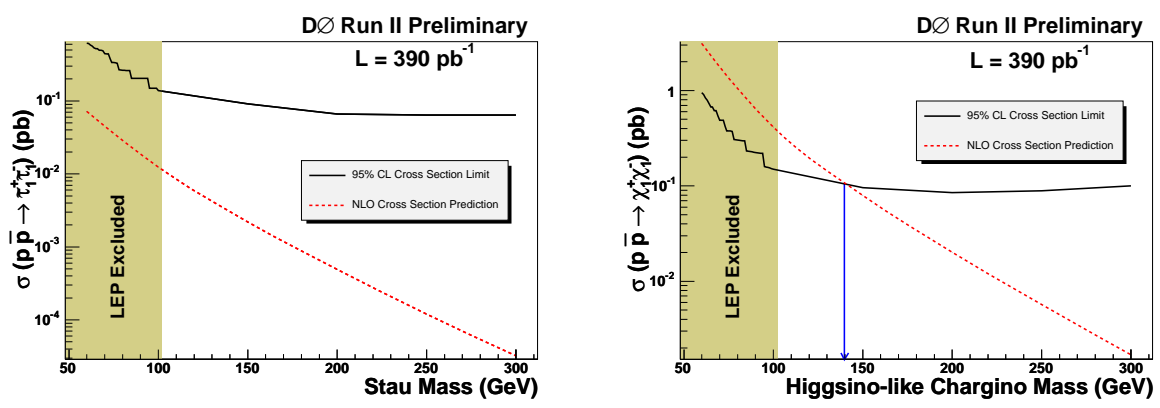

Fig. 9. Limits on pair production of GMSB stau's and AMSB chargino's from the D0 CMSP analysis.

\section{Other Analyses Under Development}

Several other analyses are underway and/or have been presented as preliminary searches for long-lived particles by the CDF and D0 collaborations. Brief descriptions of some are presented here.

$\mathrm{CDF}$ has searched for a neutral, massive, long-lived particle that decays to a $Z^{0}+X$ final state, such as a new fourth generation quark $b^{\prime} \rightarrow b Z^{0} \underline{38}$ The analysis searches for highly displaced vertices $(>0.3 \mathrm{~mm})$ with dimuon pairs whose invariant mass falls within the $Z^{0}$ mass peak window $(81<M<101 \mathrm{GeV})$. The acceptance is limited by the trigger efficiency for muons with large DCA. Separate searches are done with and without a cut of $p_{T}>30 \mathrm{GeV}$ on the $Z^{0}$ boson. Both searches observe data consistent with background and limits are set on the production cross section both as a function of mass and lifetime.

D0 has developed a new technique to search for a similar signature $\left(Z^{0}+X\right)$ in the $Z^{0} \rightarrow e^{+} e^{-}$decay mode ${ }^{39}$ By using the location of hits in the central preshower (CPS) and four layers of the electromagnetic part of the calorimeter, the incident direction of the EM object can be measured. By not requiring a reconstructed track, the analysis is sensitive to much longer lived parents. Two EM objects are used to determine a vertex position in the $x, y$ plane. The radius is measured as the distance from the PV to the EM vertex. The sign is determined by the sign of the cross product of the radius vector and the $Z^{0} p_{T}$ vector. Negative radii are used to model the background for events with positive radii. For events with an invariant mass $>75 \mathrm{GeV}$, data are consistent with the estimated background. Limits on the cross section are set. While the limits of both CDF and D0 are fairly model independent, Fig. 10] shows the preliminary limits on a long-lived $b^{\prime}$ model from both experiments.

CDF has searched for a long-lived, doubly charged Higgs boson by looking for heavily ionizing tracks $\frac{40}{4 \pm \pm}$ tracks will produce a signal several times larger than for a minimum ionizing particle in the central outer tracker (COT). One search looked for such signals while a complementary search also required large energy deposition in the calorimeter. In both searches, the expected background was $<$ 


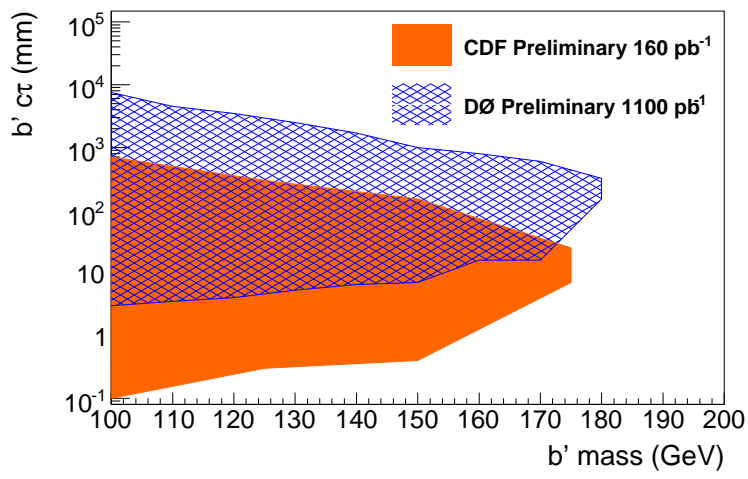

Fig. 10. Limits on the $b^{\prime}$ model from CDF (red) and D0 (blue).

$10^{-4}$ events and zero events were observed in data. In the case of $H_{L}^{ \pm \pm}$and $H_{R}^{ \pm \pm}$ degenerate in mass, a limit of $H^{ \pm \pm}<146 \mathrm{GeV}$ was set.

The full capabilities of the $\mathrm{CDF}$ and $\mathrm{D} 0$ experiments have yet to be realized. The differences between the detectors have allowed for a variety of search techniques looking for long-lived particles. Other Tevatron searches are possible including kinked tracks and detached vertices using jets. Combined with the larger data samples already recorded by the two experiments, additional results can be expected within the coming years.

\section{Summary}

The CDF and D0 collaborations have performed a wide ranging program of searches for long-lived particles that might arise from new physics. Table 2 summarizes the types of analyses and size of data samples used for each analysis. These searches greatly expand the discovery potential of the Tevatron and provide experience that will be useful at the LHC for similar work. While new limits have been set within many BSM models (as discussed above), the greatest advantage of these analyses is the ability to explore new areas that may be missed by traditional searches. The lifetimes explored cover many orders of magnitude.

In addition to the new data being accumulated at the Tevatron, new techniques are being developed to continue the exploration of additional possibilities. The indirect evidence for physics beyond the SM is overwhelming, but the lack of direct evidence drives our need to look simultaneously in many new directions in the hope that at least one will be fruitful. The Fermilab program is doing an excellent job in this regard. 
Table 2. Summary of Tevatron searches for long-lived particles.

\begin{tabular}{lccc}
\multicolumn{3}{c}{ Luminosity } \\
\hline Signal & Collaboration & $\left(\mathrm{pb}^{-1}\right)$ & Limits \\
\hline NLLP decaying to muon pairs & D0 & 380 & NuTeV excess \\
NLLP decaying to photon + MET & CDF & 570 & GMSB SUSY \\
Stopped gluinos & D0 & 410 & split supersymmetry \\
CHAMPS & CDF & 1000 & stop \\
CMSP & D0 & 390 & $\tilde{\tau}, \chi_{1}^{0}$ \\
LL $Z^{0}$ parents & CDF & 163 & $b^{\prime}$ \\
LL $Z^{0}$ parents & D0 & 1100 & $b^{\prime}$ \\
LL $H^{ \pm \pm}$ & CDF & 292 & charged Higgs \\
\hline
\end{tabular}

\section{Acknowledgments}

The author wishes to thank the CDF and D0 collaborations for producing, publishing and making available the information and plots used throughout this review. We'd like to thank Jill Adams for careful reading and Harrison Prosper for his insightful comments. This work is supported in part by the U.S. Department of Energy.

\section{References}

\section{References}

1. R. Barbier et al., Phys. Rept. 420, 1 (2005).

2. J. Abdallah et al. [DELPHI Collaboration], Eur. Phys. J. C 36, 1 (2004), erratum Eur. Phys. J. C 37, 129 (2004).

3. G. Abblendi et al. [OPAL Collaboration], Eur. Phys. J. C 33, 149 (2004).

4. A. Heister et al. [ALEPH Collaboration], Eur. Phys. J. C 31, 1 (2003).

5. P. Achard et al. [L3 Collaboration], Phys. Lett. B 524, 65 (2002).

6. V. M. Abazov et al. [D0 Collaboration], Phys. Lett. B 638, 441 (2006).

7. A. Abulencia et al. [CDF Collaboration], Phys. Rev. Lett. 98, 131804 (2007).

8. J. L. Feng and T. Moroi, Phys. Rev. D 58, 035001 (1998).

9. J. F. Gunion and S. Mrenna, Phys. Rev. D 62, 015002 (2000).

10. N. Arkani-Hamed, S. Dimopoulos, G. F. Giudice, and A. Romanino, Nucl. Phys. B709, 3 (2005).

11. L. Pape and D. Treille, Rep. Prog. Phys. 69, 2843 (2006).

12. G. R. Farrar and P. Fayet, Phys. Lett. 76B, 575 (1978).

13. H. Baer, K. m. Cheung and J. F. Gunion, Phys. Rev. D 59, 075002 (1999).

14. M. J. Strassler and K. M. Zurek, Phys. Lett. B 651, 374 (2007).

15. M. J. Strassler and K. M. Zurek, arXiv:hep-ph/0605193

16. M. J. Strassler, arXiv:hep-ph/0607160

17. T. Adams et al. [NuTeV Collaboration], Phys. Rev. Lett. 87, 041801 (2001).

18. A. Dedes, H. K. Dreiner, and P. Richardson, Phys. Rev. D 65, 015001 (2002).

19. D. Acosta et al. [CDF Collaboration], Phys. Rev. D 71, 032001 (2005).

20. V. M. Abazov et al. [D0 Collaboration], Nucl. Instrum. Meth. A 565, 463 (2006).

21. V. M. Abazov et al. [D0 Collaboration], Phys. Rev. Lett. 97, 161802 (2006).

22. L. Borissov, J. M. Conrad, and M. Shaevitz, arXiv:hep-ph/0007195

23. A. Abulencia et al. [CDF Collaboration], Phys. Rev. Lett. 99, 121801 (2007).

24. C.-H. Chen and J.F. Gunion, Phys. Rev. D 58, 075005 (1998). 
25. M. Goncharov et al., Nucl. Instrum. Methods Phys. Res., Sect. A 565, 543 (2006).

26. D. A. Toback and P. Wagner, Phys. Rev. D 70, 114032 (2004).

27. A. Heister et al. [ALEPH Collaboration], Eur. Phys. J. C 25, 339 (2002).

28. V. M. Abazov et al. [D0 Collaboration], Phys. Rev. Lett. 99, 131801 (2007).

29. J. Abdallah et al. [DELPHI Collaboration], Eur. Phys. J. C 27, 153 (2003);

30. M. Byrne, C. F. Kolda and P. Regan, Phys. Rev. D 66, 075007 (2002).

31. F. Takayama, arXiv:0704.2785 [hep-ph].

32. M. Drees and X. Tata, Phys. Lett. B 252, 695 (1990).

33. D. Acosta et al. [CDF Collaboration], Phys. Rev. Lett. 90, 131801 (2003).

34. M. Fairbairn, A. C. Kraan, D. A. Milstead, T. Sjostrand, P. Skands and T. Sloan, Phys. Rept. 438, 1 (2007).

35. http://www-cdf.fnal.gov/physics/exotic/exotic.html, CDF Note 8701.

36. W. Beenakker, M. Kramer, T. Plehn, M. Spira and P. M. Zerwas, Nucl. Phys. B 515, 3 (1998).

37. http://www-d0.fnal.gov/Run2Physics/WWW/results/np.htm, D0 Note 4746-CONF.

38. http://www-cdf.fnal.gov/physics/exotic/exotic.html, CDF Note 7244.

39. http://www-d0.fnal.gov/Run2Physics/WWW/results/np.htm, D0 Note 5454-CONF.

40. D. E. Acosta et al. [CDF Collaboration], Phys. Rev. Lett. 95, 071801 (2005). 